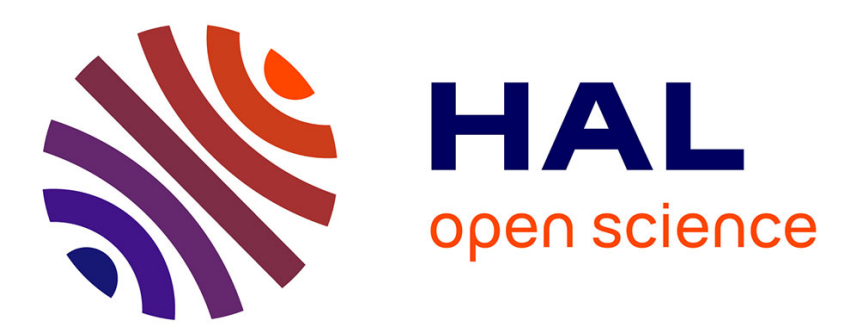

\title{
A multiscale approach to decompose a digital curve into meaningful parts
}

\author{
Thanh Phuong Nguyen, Isabelle Debled-Rennesson
}

\section{To cite this version:}

Thanh Phuong Nguyen, Isabelle Debled-Rennesson. A multiscale approach to decompose a digital curve into meaningful parts. 20th International Conference on Pattern Recognition - ICPR 2010, Aug 2010, Isatanbul, Turkey. pp.1072-1075. inria-00544733

\section{HAL Id: inria-00544733 \\ https://hal.inria.fr/inria-00544733}

Submitted on 10 Dec 2010

HAL is a multi-disciplinary open access archive for the deposit and dissemination of scientific research documents, whether they are published or not. The documents may come from teaching and research institutions in France or abroad, or from public or private research centers.
L'archive ouverte pluridisciplinaire HAL, est destinée au dépôt et à la diffusion de documents scientifiques de niveau recherche, publiés ou non, émanant des établissements d'enseignement et de recherche français ou étrangers, des laboratoires publics ou privés. 


\title{
A multi-scale approach to decompose a digital curve into meaningful parts *
}

\author{
Thanh Phuong NGUYEN and Isabelle DEBLED-RENNESSON \\ LORIA Nancy, Campus Scientifique - BP 23954506 Vandouvre-lès-Nancy Cedex, France \\ $\{$ nguyentp,debled $\} @$ loria.fr
}

\begin{abstract}
A multi-scale approach is proposed for polygonal representation of a digital curve by using the notion of blurred segment and a split-and-merge strategy. Its main idea is to decompose the curve into meaningful parts that are represented by detected dominant points at the appropriate scale. The method uses no threshold and can automatically decompose the curve into meaningful parts.
\end{abstract}

\section{Introduction}

Shape representation is an important step for many applications in computer vision such as machine recognition, shape matching. Straight lines, arcs, dominant points,... are often used to construct a good descriptor of a curve.

There are two principal objectives that are in conflict. The first is a simplification of the representation of overall shape, the second is a high precision detection of some main characteristics that may be straight line segments, corner points, ... One approach in literature for this problem is the multi-scale approach that investigate the shape representation through different unknown scales to determine a good description of the shape. Starting from Witkin's work [16], Mokhtarian [8] used curvature scale space for shape representation through multiscale. Latecki [6] used discrete curve evolution by removing vertex with minimal relevance. Kolesnikov [5] proposed an optimal split method for multi-resolution polygonal approximation of digital curves. Another approach for multi-order polygonal approximation was presented in [3]. Nguyen et al. proposed a parameter-free method [11] for polygonal representation based on dominant point detection by considering a multi-width framework to determine the most appropriate parameter.

In this paper, we present a novel approach for multi-order polygonal approximation. By applying the dominant point detector [10], a split-and-merge strategy will be used to partition a possibly noisy curve into a set of meaningful parts in which each part is represented at the appropriate scale. The rest of this paper is organized as follows. The next section recalls our previous method. Section 3 proposes a novel method for multi-resolution polygonal approximation. Sections 4 and 5 introduce some experimental results and conclusions.

*This work is supported by the ANR in the framework of the GEODIB project, BLAN06-2 134999.

\section{Dominant point detector with a fixed parameter}

In this section, we recall a method for dominant point detection that was proposed by Nguyen et Debled [10]. It uses width of blurred segments as input parameter.

\subsection{Blurred segment}

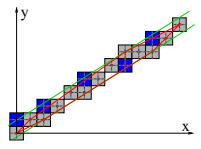

(a) A blurred segment

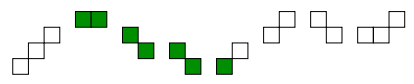

(b) A maximal blurred segment of width 1 (in dark gray points).
Figure 1. Blurred segments of width $\nu$.

The notion of blurred segment [2] is introduced from the notion of discrete line. A Discrete Line with main vector $(b, a)$, lower bound $\mu$ and thickness $\omega$, noted $D(a, b, \mu, \omega)$, is a set of points $(x, y) \in \mathbb{Z}^{2}$ that verifies: $\mu \leq a x-b y<\mu+\omega$. A Blurred Segment [2] is a set of integer points $(x, y)$ that is optimally bounded (see [2] for more detail) by the discrete line $D(a, b, \mu, \omega)$. The value $\nu=\frac{\omega-1}{\max (|a|,|b|)}$ is called the width of this blurred segment. Figure 1.a shows a blurred segment (the sequence of gray points) of which the optimal bounding line is $\mathcal{D}(5,8,-8,11)$, the vertical distance is 1.25 . Nguyen et al. proposed in [9] the notion of maximal blurred segment. A Maximal Blurred Segment of width $\nu$ (MBS) (see Fig. 1.b) is a blurred segment of width $\nu$ that cannot be extended neither at the right side nor at the left side.

\subsection{Dominant point detection}

Some criteria were proposed in [10] to locate and eliminate weak candidates of Dominant Point (DP). Let us assume a given width $\nu$, we have:

Proposition 1 A DP must be in a common zone of successive Maximal Blurred Segments (see Fig. 2.a). 


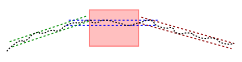

(a) Gray zone isn't a common zone

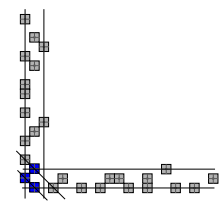

(b) Common zone in black

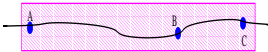
most 2 candidate of DPs (c) a MBS contain at

Figure 2. MBS and dominant point.

Proposition 2 The smallest common zone of successive Maximal Blurred Segments whose slopes are monotone contains a candidate of DP (see Fig. 2.b).

Proposition 3 A Maximal Blurred Segment contains a maximum of 2 DP candidates (see Fig. 2.c).

Heuristic strategy: In each smallest zone of successive Maximal Blurred Segments whose slopes are increasing or decreasing, the candidate as dominant point is detected as the middle point of this zone.

Based on the above study, Nguyen et al. proposed a method for the dominant point detection (see algo. 1 of [10]).

\section{Evaluation criteria}

There're 2 criteria $[14,15]$ that're used popularly for dominant point detection and polygonal approximation. Sarkar's criterion is proposed based on the error approximation and compression ratio. The error approximation may be the integral square error (ISE) that measures the quadratic error or $L_{\infty}$ that measures maximal distance between the points of input curves and the approximating polygon. The compression ratio measures the capacity of data reduction between input curve and approximating polygon. $C R=\frac{N}{N_{D P}}$. A high compression ratio leads to a high approximation error, and maintains a low approximation causes a low compression ratio. So, Sarkar [15] introduced the criterion FOM (Figure Of Merit) as ratio between compression ratio and error approximation: $F O M=\frac{C R}{I S E}$. This measure aims at balancing a high compression ratio and a low approximation error that were obtained with the dominant point detector algorithm.

Rosin [14] split the assessment into 2 components: efficiency and fidelity. The principle idea is to compare the suboptimal polygon that corresponds to dominant point detector with corresponding optimal polygon in the same conditions. The efficiency measures compression capacity of the suboptimal polygon in the same error approximation (ISE) and the fidelity measures error of the suboptimal polygon in the same number of detected dominant points: Efficiency $=$ $\frac{N_{\text {opt }}}{N_{\text {approx }}}$, Fidelity $=\frac{E_{\text {opt }}}{E_{\text {approx }}}$, where $N_{\text {opt }}$ and $N_{\text {approx }}$ are numbers of detected dominant points by using optimal and suboptimal polygon algorithm with the same error approximation; $E_{\text {opt }}$ and $E_{\text {approx }}$ are integral square errors (ISE) of error approximation by using optimal and suboptimal polygon algorithm with the same number of detected dominant point points. A combined measure is defined as geometric mean of 2 measures: Merit $=\sqrt{\text { Fidelity } \times \text { Efficiency }}$

Concerning the evaluation criteria, the merit measure allows to compare among results obtained by dominant point

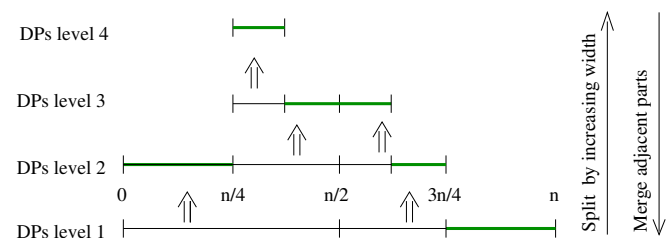

Figure 3. Multi-scale approach based on a split-and-merge strategy.

detectors in which the number of dominant point are different. However, since the complexity of a optimal algorithm is slow (see [12] for min- $\varepsilon$ problem, [13] for min-\# problem), the using of Rosin's criterion leads to an inefficient method that works in the multi-scale context. So, we propose to use an approach based on Sarkar's criterion.

Some authors $[1,7]$ argued that FOM criterion isn't sufficient for balancing the trade-off between the integral square error and the compression ration. The reason is the ISE changes rapidly than the $\mathrm{CR}$ for almost test shapes. Marji et al. [7] proposed a modified version of Sarkar: $F O M_{n}=\frac{C R^{n}}{I S E}$. In practice, they used $n=2$. In [11], Nguyen and DebledRennesson has proposed a new evaluation criterion to adapt to noisy curve: $N D^{\prime} s F O M=\frac{C R^{3}}{I S E * L_{\infty}}$. This criterion is introduced by the observation that the decision of human observer as corner depends also to local approximation. Other hand, the ISE that defines error approximation at global view, $L_{\infty}$ measure error approximation at local level. Moreover, because of the addition property of ISE, in many cases, the number of human observed corners and $L_{\infty}$ don't change but ISE increases considerably. Therefore they proposed $L_{\infty}$ as a component in the evaluation criterion.

\section{A multi-scale approach based on a split- and-merge strategy}

\section{Proposed method}

We solve here the following problem: for an input curve, can we decompose this curve into some meaningful parts represented by detected dominant points in which each part corresponds to its appropriate scale?

The principal idea is to partition a possibly noisy curve into meaningful parts in which each part corresponds to the most appropriate scale. An initial set of dominant points is constructed from the set of dominant points at width 1 . We apply a split procedure by using an evaluation criterion as a condition to determine the appropriate width for each part of the curve. The width is increased until the scale where the evaluation criterion reaches to a peak. The evaluation criterion can be Marji's FOM or ND's FOM. By merging the adjacent parts that have a same scale, the curve is decomposed into meaningful parts in which the dominant points are detected at the scale corresponding to this meaningful parts.

We present in algorithm 1 a procedure to split a partial curve if the evaluation criterion on the dominant point detection is better at the next width parameter in comparison with the current width value. Figure 3 illustrates how to split a 


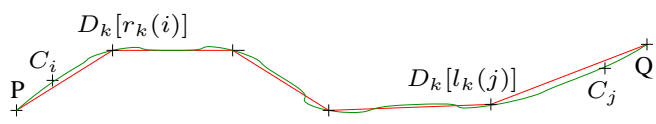

Figure 4. $E V_{k}(P, Q, i, j)$

curve into meaningful part by using this procedure. Its principal idea is to compare the evaluation criterion on the detected dominant points at the current width and the next width. If the result is higher at the next width, this partial curve will be splitted into 2 half parts to consider at higher width by applying recursively this procedure. Otherwise, this partial curve is considered as the best representation at the current width, and this procedure stops at this scale.

We use the notation $D_{k}$ (resp. $D$ ) to indicate the set of extracted dominant points at the with $k$ (resp. through multiscale approach); $D_{k}[m]$ (resp. $D[m]$ ) corresponds to the $m^{t h}$ point in the corresponding set. The notations $l_{k}(i)$ and $r_{k}(i)$ (resp. $l(i)$ and $r(i)$ ) are used to indicate the nearest index in the set of extracted dominant points at with $k$ (resp. through multi-scale approach) at the left and right sides of the $i^{t h}$ point of the input curve. In addition, the notation $E V_{k}(P, Q, i, j)$ (see also figure 4 ) is used to indicate the evaluation criterion that is determined between the part of curve from $P$ to $Q$ and the polygon constructed from 2 points $P, Q$ and the set of extracted dominant point at width $k$ between $C_{i}$ and $C_{j}(i$ and $j$ are 2 indexes in the curve between $P$ and $Q$ ).

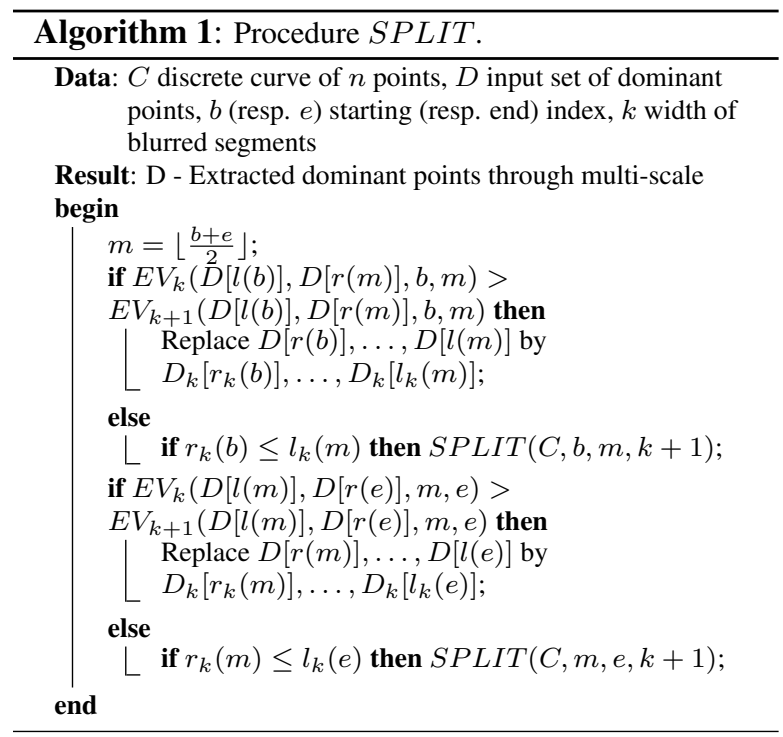

Thanks to algorithm 1, we propose in algorithm 2 a method to decompose a curve into meaningful parts with their extracted dominant points. Firstly, a sequence of dominant point sets is detected at each width from 1 to $\max W i d t h$ by using algorithm 1 of [10]. The set of dominant points at width 1 is considered as initial dominant point set. Then we apply recursively the procedure presented in algorithm 1 to determine the decomposition of curve into meaningful parts with their corresponding scale. Finally, by merging the adjacent parts that stop at a same width, we obtain the decomposition of the curve.

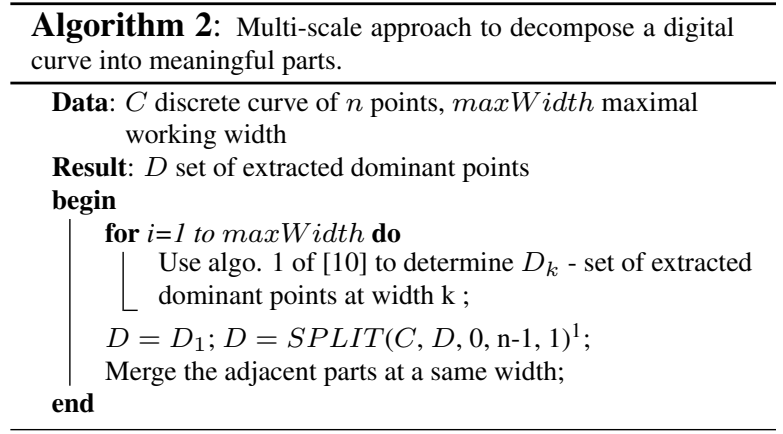

\section{Complexity}

For each part of the curve, we can use the same arguments as in [11] to conclude that the corresponding width can be seen as constant in relation with the number of points of this part. So, the maximal width can be seen also as constant in comparison with number of points of the curve. Thanks to recent result [4], the decomposition of a curve into maximal blurred segments can be done in $O(n \log n)$, the algorithm 1 of [10] can be done in $O(n \log n)$. The procedure $S P L I T$ wastes $O(n)$ time at each scale. So, the complexity of algorithm 2 is also $O(n \log n)$.

\section{Experimentation and comparison with other free-parameter methods}

Experimentation: Figure 5 shows several obtained results in which each meaningful part is represented by each color.

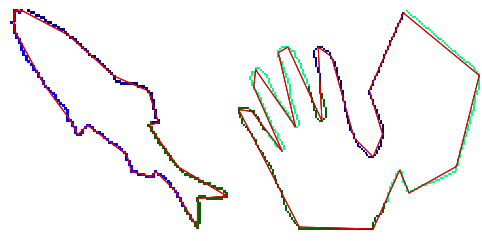

(a) (b)

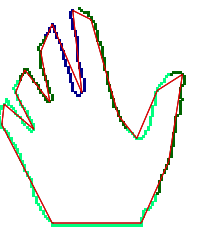

(c)

\section{Figure 5. Results that were obtained with the proposed method.}

Comparison: We present in figures 6,7 and table 1 the comparisons between this proposed method and freeparameter method [11] and multi-order method [3]. These results are considered by using two evaluation criteria: Marji's criterion and Nguyen-Debled's criterion. Table 1 shows that this proposed method gives better results than those of the free-parameter method and the multi-order one.

\section{Conclusion}

We have presented a solution for decomposition of a curve into meaningful parts with detected dominant points. Each

\footnotetext{
${ }^{1} \mathrm{D}$ contains dominant points with position and scale informations.
} 


\begin{tabular}{|c|c|c|c|c|c|c|c|c|c|c|}
\hline Curve & Figures & $n$ & Method & $n D P$ & $I S E$ & $L_{\infty}{ }^{2}$ & $C R$ & Marji's FOM & ND's FOM & Width \\
\hline \multirow{2}{*}{ Leaf } & \multirow{2}{*}{ 6.a, 6.b } & \multirow{2}{*}{370} & Proposed & 19 & 595.544 & 3.48 & 19.474 & $\mathbf{0 . 6 3 7}$ & $\mathbf{3 . 5 6 3}$ & \\
\cline { 4 - 12 } & & & Free-parameter [11] & 22 & 509.619 & 3.394 & 16.818 & 0.555 & 2.750 & 3 \\
\hline \multirow{2}{*}{ Plane } & \multirow{2}{*}{ 6.c, 6.d } & \multirow{2}{*}{523} & Proposed & 49 & 241.5 & 1.45 & 10.6735 & $\mathbf{0 . 4 7 1 7}$ & $\mathbf{3 . 4 7 2}$ & \\
\cline { 4 - 12 } & & Free-parameter [11] & 40 & 374.382 & 3.5938 & 13.075 & 0.45566 & 1.657 & 2 \\
\hline \multirow{2}{*}{ Rabbit } & \multirow{2}{*}{ 7.a, 7.b } & \multirow{2}{*}{821} & Proposed & 10 & 911.54 & 4.743 & 28.1 & $\mathbf{0 . 8 6 6}$ & $\mathbf{5 . 1 3 2}$ & \\
\cline { 4 - 12 } & & Multi-order [3] & 11 & 1663.82 & 5.514 & 25.545 & 0.3922 & 1.817 & \\
\hline
\end{tabular}

Table 1. Comparisons on the curves in figures 6,7 with [11] and [3].

meaningful part corresponds to its natural scale. Therefore, the object boundary can be represented better with detected dominants points through this multi-scale framework. The proposed method can work with possibly disconnected noisy curves thanks to the notion of blurred segment. The benefit of this method is clearly its adaptability to the noise level present in the curves if it is irregularly distributed.

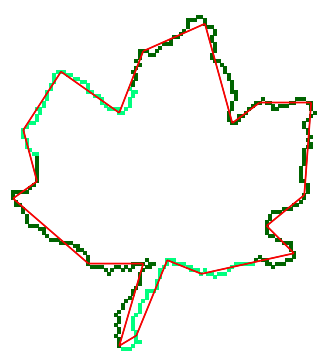

(a) Multi-scale

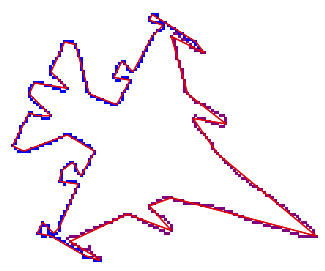

(c) Multi-scale

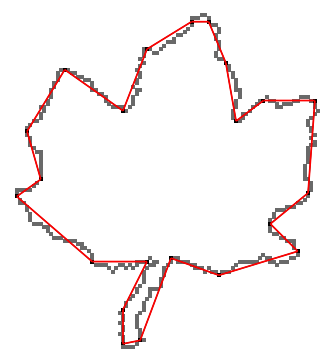

(b) Free-parameter

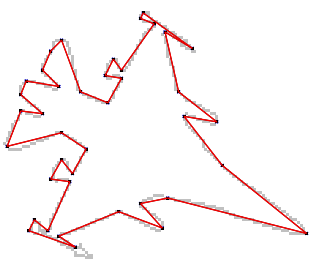

(d) Free-parameter
Figure 6. Comparison with [11].

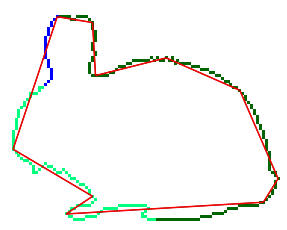

(a) Multi-scale

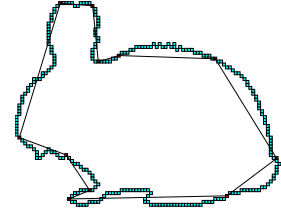

(b) Debled et al. [3]
Figure 7. Comparison with [3].

\section{References}

[1] F. Arrebola and F. S. Hernández. Corner detection and curve segmentation by multiresolution chain-code link-

\footnotetext{
${ }^{2} L_{\infty}$ : error approximation measure
}

ing. Pattern Recognition, 38(10):1596-1614, 2005.

[2] I. Debled-Rennesson, F. Feschet, and J. Rouyer-Degli. Optimal blurred segments decomposition of noisy shapes in linear time. Computers \& Graphics, 30(1), 2006.

[3] I. Debled-Rennesson, S. Tabbone, and L. Wendling. Fast polygonal approximation of digital curves. In ICPR, volume 1, pages 465-468, 2004.

[4] A. Faure and F. Feschet. Tangential cover for thick digital curves. In DGCI, volume 4992 of $L N C S$, pages 358369, 2008.

[5] A. Kolesnikov, P. Fränti, and X. Wu. Multiresolution polygonal approximation of digital curves. In ICPR, volume 2, pages 855-858, 2004.

[6] L. J. Latecki and R. Lakämper. Polygon evolution by vertex deletion. In Scale-Space, volume 1682 of LNCS, pages 398-409, 1999.

[7] M. Marji and P. Siy. Polygonal representation of digital planar curves through dominant point detection - a nonparametric algorithm. Pattern Recognition, 37(11):2113-2130, 2004.

[8] F. Mokhtarian and A. Mackworth. Scale-based description and recognition of planar curves and twodimensional shapes. PAMI, 8(1):34-43, Jan. 1986.

[9] T. P. Nguyen and I. Debled-Rennesson. On the local properties of digital curves. International Journal of Shape Modeling, 14(2):105-125, 2008.

[10] T. P. Nguyen and I. Debled-Rennesson. Detection of dominant points on noisy curves. In ICIP, pages 953956, Cairo, Egypt, November 2009.

[11] T. P. Nguyen and I. Debled-Rennesson. Free-parameter method for polygonal representation of noisy curves. In RPS, editor, IWCIA, Cancun, Mexico, November 2009.

[12] J. C. Pérez-Cortes and E. Vidal. Optimum polygonal approximation of digitized curves. Pattern Recognition Letters, 15(8):743-750, 1994.

[13] A. Pikaz and I. Dinstein. Optimal polygonal approximation of digital curves. Pattern Recognition, 28(3):373379, 1995.

[14] P. L. Rosin. Techniques for assessing polygonal approximations of curves. PAMI, 19(6):659-666, 1997.

[15] D. Sarkar. A simple algorithm for detection of significant vertices for polygonal approximation of chaincoded curves. Pattern Recognition Letters, 14(12):959964, 1993.

[16] A. P. Witkin. Scale-space filtering. In International Joint Conferences on Artificial Intelligence, pages 10191022, 1983. 